\title{
THE LENGTH OF ELEMENTS IN FREE SOLVABLE GROUPS
}

\author{
CARL DROMS, JACQUES LEWIN, AND HERMAN SERVATIUS
}

(Communicated by Ronald Solomon)

\begin{abstract}
We examine the relationship between the complexity of the word problem for a presentation and the complexity of the problem of determining the length of a shortest word equivalent to a given word. Our main result is that the length of the element represented by a word in a free solvable group can be determined in polynomial time.
\end{abstract}

Let $\mathscr{P}=\langle X \mid R\rangle$ be a group presentation. Let $F$ be free on $X$, let $N$ be the normal closure of $R$ in $F$, and let $G=F / N$ be the group presented by $\mathscr{P}$. A word over $X$ is a finite string of symbols $x$ and $x^{-1} \quad(x \in X)$. The length of $a$ word $w$ is the length of $w$ regarded as a string. Clearly, each word over $X$ represents a unique element of $G$. The length of an element $g \in G$ is defined to be the number of symbols in any shortest word representing $g$.

Let $\mathscr{C}$ be the Cayley graph of $G$ with respect to the presentation $\mathscr{P}$; that is, $\mathscr{C}$ is the graph with vertex set $G$ and (directed) edge set $E=G \times X$, where the edge $e=(g, x)$ goes from $g$ to $g x$. We call $g$ the initial vertex of $e$, denoted $l(e)$, and $g x$ its terminal vertex, denoted $\tau(e)$. The generator $x$ is called the label of $e$. The length of a group element $g$ is then the distance (under the graph metric) from 1 to $g$ in $\mathscr{C}$.

The Word Problem $\mathscr{W}(\mathscr{P})$ for a presentation $\mathscr{P}$ asks for an algorithm which decides, given any word $w$ over $X$, whether $w$ represents the identity of $G$, and its Length Problem $\mathscr{L}(\mathscr{P})$ asks for an algorithm which determines, given such a word $w$, what the length of the corresponding group element is.

It is clear that $\mathscr{W}=\mathscr{W}(\mathscr{P})$ is solvable if and only if $\mathscr{L}=\mathscr{L}(\mathscr{P})$ is; if $\mathscr{L}$ is solvable, then $\mathscr{W}$ is, as well, since the identity is the only element of length 0 . In fact, $\mathscr{W}$ is no more complex than $\mathscr{L}$. On the other hand, if $\mathscr{W}$ is solvable, then so is $\mathscr{L}$; given a word $w$, one can construct, one after another, the spheres of the Cayley graph of $G$. Each time a new element $e$ is constructed, one can solve $\mathscr{W}$ for $e^{-1} w$. Since, in general, the number of elements of a given length in a group is an exponential function of the length, this solution of $\mathscr{L}$ may be exponentially more complex than the solution of $\mathscr{W}$.

In fact, the following (metabelian) example of Parry [4] shows that it is possible for a presentation to have a word problem which is solvable in polynomial time and a length problem which is $\mathscr{N} \mathscr{P}$-complete.

Received by the editors January 20, 1992.

1991 Mathematics Subject Classification. Primary 20F10. 
Example. Let $G$ be the wreath product $Z_{2} l(Z \times Z)$, given by the standard infinite presentation

$$
\left.\langle t, a, b|[a, b]=1, t^{2}=1, t^{x} t^{y}=t^{y} t^{x} \text { for each } x, y \in \operatorname{gp}\{a, b\}\right\rangle .
$$

Each element of $G$ can be expressed in the form

$$
t^{y_{1}} t^{y_{2}} \cdots t^{y_{k}} x
$$

where $x \in \operatorname{gp}\{a, b\}$ and $y_{1}, \ldots, y_{n}$ are distinct elements of $\operatorname{gp}\{a, b\}$, and this expression is unique up to the order of the conjugating elements $y_{i}$. Now it is clear that any word of length $n$ in $a, b$, and $t$ can be rewritten in this form in at most $O\left(n^{2}\right)$ steps by commuting all the $a$ 's and $b$ 's to the right of all the $t$ 's. Since two such words represent the same element of $G$ if and only if the respective lists of conjugating elements are permutations of one another and the values of " $x$ " are the same, the word problem is solvable in time $O\left(n^{2}\right)$.

On the other hand, the length problem for $G$ can be reduced to the Euclidean Traveling Salesman Problem in the integer lattice in the plane, the Cayley graph of the group $\langle a, b \mid[a, b]=1\rangle$. An element

$$
t^{y_{1}} t^{y_{2}} \cdots t^{y_{k}} x
$$

can be represented by a path in the integer lattice which starts at 1 , stops at each vertex $y_{i}$ to "light a lamp", and then proceeds to $x$ [4]. (It is for this reason that this group has been called a "Lamplighter Group" by J. Cannon.)

Finding a shortest word representing an element of the form

$$
t^{y_{1}} t^{y_{2}} \ldots t^{y_{m}}
$$

is thus equivalent to finding a shortest circuit in the integer lattice in the plane which begins at the origin and passes through each of the vertices corresponding to the $y_{i}$, a problem which is known to be $\mathscr{N} \mathscr{P}$-complete [3].

However, if a group has polynomial growth with respect to the given generators and if the word problem can be solved in polynomial time, then the length problem is solvable in polynomial time, as well. Suppose that the number of elements of length $n$ is $O\left(n^{k}\right)$ for some positive integer $k$. Then having constructed the $(n-1)$-sphere of $\mathscr{C}$, one can construct the $n$-sphere in time $O\left(n^{2 k} w(n)\right)$, where $w(n)$ is the time needed to solve the word problem for two words of length $\leq n$. Given a "standard" word $W_{g}$ of length $n-1$ representing each element $g \in G$ of length $n-1$, form the words $W_{g} a$ and $W_{g} a^{-1}$ for each generator $a$. Each of the $O\left(n^{k}\right)$ new words must be compared to the $O\left(n^{k}\right)$ standard words of length $n-1$ and $n-2$, as well as to each of the other new words, resulting in $O\left(n^{2 k}\right)$ solutions of the word problem for words of length $\leq n$. Hence, the $n$-ball of $\mathscr{C}$ can be constructed in polynomial time, and since the word problem is solvable in polynomial time, so is the length problem.

In certain cases - free groups, for example - the length problem can be solved in polynomial time even though the group grows exponentially. In fact, this is true of any hyperbolic group with exponential growth, since in this case there is a quadratic algorithm for computing geodesic representatives (see [2]).

The purpose of this note is to prove 
Theorem 1. Let $\mathscr{P}=\langle X \mid R\rangle$ be a presentation with $F$ free on $X$ and $N$ the normal closure of $R$. If the length of the element of $G=F / N$ represented by a word $w$ of length $n$ can be determined in time $O(t(n))$, then the length of the element of $G_{1}=F / N^{\prime}$ represented by $w$ can be determined in time $O\left(n^{2} t(n)\right)$.

For example, if the word problem for $F / N$ can be solved in polynomial time and $F / N$ is infinite, then $F / N^{\prime}$ has exponential growth, but its length problem can be solved in polynomial time. Similarly, if $N^{(k)}$ denotes the $k$ th derived group of $N$, then the groups $F / N^{(k)} \quad(k \geq 2)$ all have exponential growth and polynomial length problem. In particular, the word problem for the free abelian group $F / F^{\prime}$ has a word problem solvable in linear time, so we have

Corollary 1. If $F$ is freely generated by the finite set $X$, and if $F^{(k)}$ denotes the $k$ th derived group of $F$, then the length of the element of $F / F^{(k)}$ represented by a word over $X$ of length $n$ can be determined in time $O\left(n^{2 k-1}\right)$.

Let $\mathscr{C}$ be the Cayley graph of $F / N$. Then the fundamental group of $\mathscr{C}$ is naturally isomorphic to $N$-the label of any edge path in $\mathscr{C}$ can be interpreted as an element of $F$, and the path is closed precisely when this element belongs to $N$. Thus, the abelianized group $N / N^{\prime}$ is the first homology of $\mathscr{C}$, so a path $P$ in $\mathscr{C}$ represents an element of $N^{\prime}$ if and only if it is homologically trivial.

Given a word $w$ over $X$, let $\bar{w}$ denote the element of $G=F / N$ represented by $w$ and let $P_{w}$ denote the edge-path in $\mathscr{C}$ beginning at the vertex 1 and labeled $w$. Note that $\bar{w}$ is the ending vertex of $P_{w}$. We will define two functions $\varepsilon_{w}: E \rightarrow \mathbb{Z}$ and $\nu_{w}: V \rightarrow \mathbb{Z}$, respectively the "edge numbering" and the "vertex numbering" associated to the path $P_{w}$.

For each edge $(g, x) \in E=G \times X$, we set $\varepsilon_{w}(g, x)$ equal to the net number of times the edge $(g, x)$ is traversed by $P_{w}$-each traversal from $g$ to $g x$ counting +1 and each traversal from $g x$ to $g$ counting -1 . Since $\mathscr{C}$ is a one-dimensional complex, $P_{w}$ represents the trivial homology class in $H_{1}(\mathscr{C})$ if and only if $\varepsilon_{w}$ is identically 0 .

Given two words $w$ and $u$, a straightforward computation shows that, for any edge $(g, x)$ of $\mathscr{C}$,

$$
\varepsilon_{w u^{-1}}(g, x)=\varepsilon_{w}(g, x)-\varepsilon_{u}\left(\overline{u w}^{-1} g, x\right) .
$$

For each vertex $g \in V=G$, define $\nu_{w}(g)$ equal to the net number of "entrances into" the vertex $g$ of $\mathscr{C}$ along the path $P_{w}$; that is,

$$
\nu_{w}(g)=\sum_{g=\tau(e)} \varepsilon_{w}(e)-\sum_{g=\imath(e)} \varepsilon_{w}(e) .
$$

(Note in particular that $\nu_{w}$ is completely determined by $\varepsilon_{w}$.)

It is clear that if $w$ is any word, then $\nu_{w}$ satisfies "Kirchhoff's Law", that is, either $\nu_{w}$ is identically 0 (if $\bar{w}=1$ ), or $\nu_{w}(1)=-1, \nu_{w}(\bar{w})=+1$, and $\nu_{w}(h)=0$ for each other element $h \in G($ if $\bar{w} \neq 1)$.

Lemma 1. Two words $w$ and $u$ over $X$ represent the same element of $G_{1}=$ $F / N^{\prime}$ if and only if the functions $\varepsilon_{w}$ and $\varepsilon_{u}$ are identically equal.

Proof. If $w$ and $u$ are equivalent $\bmod N^{\prime}$, then $\bar{w}=\bar{u}$ and $\varepsilon_{u u^{-1}} \equiv 0$. Thus, by (1), $\varepsilon_{w} \equiv \varepsilon_{u}$. 
Conversely, if $\varepsilon_{w} \equiv \varepsilon_{u}$, then $\bar{w}=\bar{u}$, since the edge paths $P_{w}$ and $P_{u}$ must end at the same point. Therefore, $\varepsilon_{w u^{-1}} \equiv 0$, by (1), so $P_{w u^{-1}}$ represents $0 \in H_{1}(\mathscr{C})$. That is to say, $w \equiv u\left(\bmod N^{\prime}\right)$.

Let $n: E \rightarrow \mathbb{Z}$ be an arbitrary integer-valued function on the edge set $E$ of $\mathscr{C}$. Then we can define a function $\bar{n}: G \rightarrow \mathbb{Z}$ by setting

$$
\bar{n}(g)=\sum_{g=\tau(e)} n(e)-\sum_{g=l(e)} n(e) .
$$

We will say that the function $n$ is geometric if $\sum_{e \in E}|n(e)|<\infty$ and the associated function $\bar{n}$ on $G$ satisfies Kirchhoff's Law. If $n$ is geometric and $\bar{n}$ is zero, we will say $n$ is closed, otherwise $n$ is open.

It is clear that if $w$ is any word over $X$, then $\varepsilon_{w}$ is geometric, $\overline{\varepsilon_{w}}=\nu_{w}$, and $\varepsilon_{w}$ is closed if and only if $\bar{w}=1$. Conversely, given an arbitrary edge numbering $n: E \rightarrow \mathbb{Z}$, we shall see that there is a word $w$ over $X$ with $\varepsilon_{w}=n$ if and only if $n$ is geometric and that the corresponding edge path $P_{w}$ is closed if and only if $n$ is closed. Any word $w$ with $\varepsilon_{w}=n$ will be said to realize $n$ (and we remark that $w$ is not unique).

Given $n: E \rightarrow \mathbb{Z}$, let $\operatorname{supp}(n)$ denote the subgraph of $\mathscr{C}$ consisting of all edges $e$ with $n(e) \neq 0$, together with their end points, and let $\operatorname{supp}^{+}(n)$ denote the graph $\operatorname{supp}(n) \cup\{1\}$.

Lemma 2. Let $n$ be a geometric numbering on $\mathscr{C}$ with $\operatorname{supp}(n)$ connected.

(1) If $n$ is closed, then, for each $v \in \operatorname{supp}(n)$, there is a loop in $\mathscr{C}$ based at $v$ which traverses each edge e exactly $n(e)$ times.

(2) If $n$ is open, then $1 \in \operatorname{supp}(n)$, and there is a path in $\mathscr{C}$ beginning at 1 which traverses each edge e exactly $n(e)$ times.

In particular, no edge is traversed in both directions by such a path.

Proof. The proof of the lemma is essentially the same as the proof that any connected graph in which the vertex degrees are all even possesses an Euler circuit.

Note that the length of any such path is $\sum_{e \in E}|n(e)|$.

Let $n$ be a geometric (closed or open) numbering on $E$. Let $s_{1}, s_{2}, \ldots, s_{r}$ be the connected components of $\operatorname{supp}^{+}(n)$, where $s_{1}$ is the component containing 1. It is clear that if $n$ is open, then the unique vertex $v$ with $\bar{n}(v)=+1$ lies in $s_{1}$.

For each $i, j \leq r$, let $d_{i j}=d_{j i}$ be the distance in $\mathscr{C}$ from $s_{i}$ to $s_{j}$, and let $p_{i j}$ be a path in $\mathscr{C}$ from $s_{i}$ to $s_{j}$ of length $d_{i j}$, where, for convenience, we take $p_{j i}$ to be the inverse of $p_{i j}$. Let $v_{i j}$ be the initial point of $p_{i j}$ so that the terminal point of $p_{i j}$ is $v_{j i}$. (See Figure 1.)

Let $K_{r}$ be a complete undirected graph with vertices $k_{1}, \ldots, k_{r}$ in 1-1 correspondence with the $s_{i}$, and assign the weight $d_{i j}$ to the edge joining $k_{i}$ and $k_{j}$. Let $T(n)$ be a minimal-weight spanning tree for $K_{r}$, and let $W(n)$ be the sum of the weights of the edges of $T(n)$-note that, while $T(n)$ is not uniquely determined by $n, W(n)$ is. It is clear that any path in $\mathscr{C}$ which realizes $n$ must have length at least $\sum_{e \in E}|n(e)|+2 W(n)$. Any such path having this length will be called a minimal realization of $n$.

Lemma 3. If $n: E \rightarrow \mathbb{Z}$ is geometric, then there is a minimal realization of $n$ beginning at the vertex 1 in $\mathscr{C}$. 


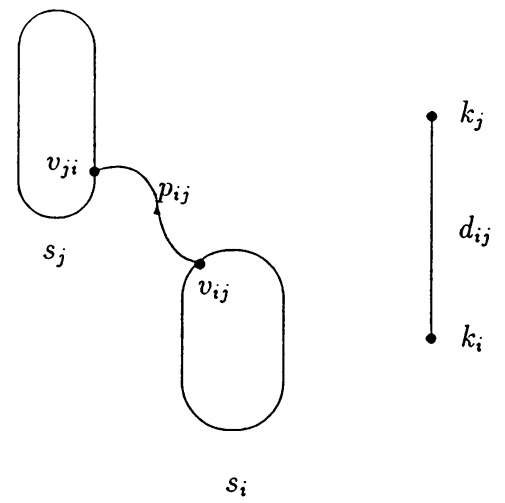

FIGURE 1

Proof. The proof is by induction on $r$, the number of components of $\operatorname{supp}^{+}(n)$. If $r=1$, the result follows from Lemma 2 .

If $r>1$, choose a pendent vertex $k_{j}$ of $T(n)$ such that $1 \notin s_{j}$, and let $k_{i}$ be the vertex of $T(n)$ to which $k_{j}$ is adjacent. Define new integer-valued functions $n^{\prime}$ and $n^{\prime \prime}$ on $E$ as follows:

$$
\begin{aligned}
& n^{\prime}(e)= \begin{cases}n(e) & \text { if } e \notin s_{j}, \\
0 & \text { otherwise }\end{cases} \\
& n^{\prime \prime}(e)= \begin{cases}n(e) & \text { if } e \in s_{j}, \\
0 & \text { otherwise }\end{cases}
\end{aligned}
$$

It is clear that $n^{\prime}$ and $n^{\prime \prime}$ are geometric, $n^{\prime \prime}$ is closed, $\operatorname{supp}\left(n^{\prime \prime}\right)=s_{j}$, and $\operatorname{supp}^{+}\left(n^{\prime}\right)$ has $r-1$ components. Furthermore, $W\left(n^{\prime}\right)=W(n)-d_{i j}$. Thus, there is a minimal realization $p^{\prime}$ of $n^{\prime}$ beginning at 1 , whose length is

$$
\sum_{e \in E}\left|n^{\prime}(e)\right|+2\left(W(n)-d_{i j}\right)
$$

The vertex $v_{i j}$ lies on the path $p^{\prime}$. To get a path which realizes $n$, follow $p^{\prime}$ until $v_{i j}$, follow $p_{i j}$ to $v_{j i} \in s_{j}$, follow a minimal realization of $n^{\prime \prime}$ beginning and ending at $v_{j i}$ (which exists by Lemma 2, since $n^{\prime \prime}$ is closed), follow $p_{i j}^{-1}$ back to $v_{i j}$, and then proceed along $p^{\prime}$. Clearly, the length of this path is $\sum_{e \in E}|n(e)|+2 W(n)$, so it is a minimal realization of $n$.

Theorem 2. Let $w$ be a word over $X$. Then the length of the element of $G_{1}=$ $F / N^{\prime}$ spelled " $w$ " is $\sum_{e \in E}\left|\varepsilon_{w}(e)\right|+2 W\left(\varepsilon_{w}\right)$.

\section{THE ALGORITHM}

In this section we will prove Theorem 1, which we restate here for convenience.

Theorem 1. Let $\mathscr{P}=\langle X \mid R\rangle$ be a presentation with $F$ free on $X$ and $N$ the normal closure of $R$. If the length of the element of $G=F / N$ represented by a word $w$ of length $n$ can be determined in time $O(t(n))$, then the length of the element of $G_{1}=F / N^{\prime}$ represented by $w$ can be determined in time $O\left(n^{2} t(n)\right)$. Proof. Let $\mathscr{C}$ denote the Cayley graph of $G$ corresponding to the presentation $\mathscr{P}$. We will describe an algorithm to solve the length problem for $G_{1}$. 
Let $w$ be a word over $X$ of length $n$ and let $p$ be the path at 1 labeled $w$. For $i=1,2, \ldots, n$, let $w_{i}$ be the $i$ th letter of $w$ and let $w^{i}$ be the $i$-prefix of $w$; that is, $w^{i}=w_{1} w_{2} \cdots w_{i}$. Let $v_{1}, v_{2}, \ldots, v_{k}$ be the vertices visited by $p$. (Note that $k$ will, in general, be $<n$, since $p$ may visit a single vertex more than once.)

In the first part of the algorithm, we will scan $w$ from left to right and construct two matrices: $D$, whose $i j$-entry will be the distance in $\mathscr{C}$ from the vertex $v_{i}$ to the vertex $v_{j}$, and $S$, whose $i j$-entry is the net number of traversals of the edge from $v_{i}$ to $v_{j}$ ( 0 if $v_{i}$ and $v_{j}$ are not adjacent.) Note that $D$ is symmetric and that, for $i \neq j$, the $i j$-and $j i$-entries of $S$ are additive inverses.

Suppose that we have read the prefix $w^{i-1}$ and that the matrices $S$ and $D$ have been constructed for the word $w^{i-1}$. Let $1=v_{1}, v_{2}, \ldots, v_{l}$ be the vertices visited by the path at 1 labeled $w^{i-1}$ in $\mathscr{C}$, and let $v$ be the end vertex of the path at 1 labeled $w^{i}$. At the $i$ th step, we read $w_{i}$ and

(1) solve $\mathscr{W}$ for $\mathscr{P} l$ times to see if the current vertex $v$ is new;

(2) if $v$ is new, set $v_{l+1}=v$, add row $l+1$ and column $l+1$ to $D$, and compute the entries in the new row and column by repeatedly solving the length problem for $\mathscr{P}$;

(3) if $v$ is new, add row $l+1$ and column $l+1$ to $S$, and set their entries to 0 ;

(4) add or subtract 1 to the appropriate entries in $S$ to reflect the fact that the edge from $v_{j}$ to $v$ has been traversed \pm 1 times more.

Note that for each of the $n$ iterations, the first two steps require at most $O(n t(n))$ steps each, the third requires at most $O(n)$, and the fourth a constant number of steps. Thus, the first part of the algorithm can be accomplished in time at most $O\left(n^{2} t(n)\right)$.

In the second part of the algorithm, we

(1) identify the connected components of the graph with incidence matrix $M$ obtained by setting each nonzero entry of $S$ to 1 ;

(2) compute the distances in $\mathscr{C}$ between these connected components, by examining $D$;

(3) identify a minimal spanning tree for the complete graph whose edge weights are the numbers computed in the previous step.

Here, each step requires at most $O\left(n^{2}\right)$ steps (see, e.g., [1]). Thus, the whole algorithm requires time at most $O\left(n^{2} t(n)\right)$.

Corollary 1 now follows from the fact that the length problem can be solved for $F / F^{\prime}=F / F^{(1)}$ in time $O(n)$.

\section{REFERENCES}

1. A. Aho, J. Hopcroft, and J. Ullman, The design and analysis of computer algorithms, Addison-Wesley, Reading, MA, 1974.

2. D. B. A. Epstein, J. W. Cannon, D. F. Holt, S. V. F. Levy, M. S. Patterson, and W. P. Thurston, Word processing in groups, Jones and Bartlett, Boston and London, 1992.

3. C. H. Papadimitriou, The Euclidean traveling salesman problem is $\mathscr{N} \mathscr{P}$-complete, Theoret. Comput. Sci. 4 (1977), 237-244.

4. W. Parry, Growth series of some wreath products, preprint. 
Department of Mathematics, James Madison University, Harrisonburg, Virginia 22807

E-mail address, C. Droms: carl@cayuga.cs.jmu.edu

Department of Mathematics, Syracuse University, Syracuse, New York 13244

E-mail address, J. Lewin: jlewin@suvm.acs.syr.edu

Department of Mathematics, Massachusetts institute of Technology, Cambridge, MASSACHUSETTS 02139

Current address, H. Servatius: Department of Mathematics, Tufts University, Medford, Massachusetts 02155

E-mail address, H. Servatius: hservati@jade.tufts.edu 